\title{
Effect of tapered FRP sheets on interlaminar fracture behaviour of FRP-concrete interface
}

\author{
Bo Gao ${ }^{\mathrm{a}}$, Jang-Kyo Kim ${ }^{\mathrm{a} *}$ and Christopher K. Y. Leung ${ }^{\mathrm{b}}$ \\ ${ }^{\mathrm{a}}$ Department of Mechanical Engineering and ${ }^{\mathrm{b}}$ Department of Civil Engineering \\ Hong Kong University of Science \& Technology \\ Clear Water Bay, Hong Kong
}

\begin{abstract}
External bonding of fiber reinforced plastic (FRP) sheets to reinforced concrete (RC) beams has been accepted as an effective strengthening method. The use of taper ended FRP sheets can further enhance the strengthening performance, improving both the load carrying capacity and the corresponding deflection beyond those of the beams bonded with FRP sheets of a constant thickness. This paper presents experimental and analytical investigations regarding the influence of tapered FRP sheets on interlaminar fracture resistance between the FRP and concrete. The asymmetric double cantilever beam (ADCB) test was used to measure the interlaminar fracture resistance. The results showed that the taper configuration could significantly affect the interlaminar fracture behavior in terms of both the load-displacement behavior and the variation of critical strain energy release rate with crack propagation.
\end{abstract}

Keywords: Interlaminar fracture toughness; Fibers; Taper.

*Corresponding author. Tel: 852-2358 7207; Fax: 852-2358 1543; Email: mejkkim@ust.hk 


\section{Introduction}

With growing needs for strengthening and rehabilitation of concrete structures, the external bonding of fibre reinforced plastic (FRP) sheets to the reinforced concrete (RC) structure has attracted significant research in the past decade [1-7]. These studies found that the FRP reinforcement significantly increased the ultimate load carrying capacity of RC beams. However, the improvement was at the expense of the ductility of the beams due to the premature failure modes, such as concrete cover separation, which prevented the full utilization of the tensile strength of the FRP sheet. In an effort to delay the concrete cover separation and thus further enhance the strengthening performance, tapered FRP sheets were applied, where the FRP sheet thickness was varied gradually near the cut-off points.

The successful applications and long-term durability of the FRP strengthened concrete structure depend largely on the reliable bonding between the FRP sheets and concrete [8]. Various interfacial debonding failures and concrete cover separation arising from the stress concentrations at the locations of flexure and shear/flexural cracks or at the ends of FRP sheets have been identified as the major premature failure modes $[9,10]$. Sufficiently high interlaminar fracture resistance is essential to preventing the premature failures from taking place.

This paper aims to study the interlaminar fracture resistance of FRP sheetadhesive-concrete joints with different tapered end geometries using the asymmetric double cantilever beam (ADCB) test. Following our recent work [11] on the interlaminar fracture toughness of FRP-concrete interface bonded with rubber-modified epoxy and vinylester resins, the ADCB test was applied. Closed-form solutions were developed to calculate the critical strain energy release rates of the ADCB specimens 
with tapered FRP sheets based on the elastic beam theory and the calibration method. From both the fundamental and practical view points, these formulas are of particular relevance to many situations where tapered geometries are used, such as FRP repair patches of aerospace and marine structures. Special emphasis was placed on identification of the changes in load-displacement curves and the study of the crack growth resistance behaviours between different taper geometries, especially near the taper where the FRP sheet thickness changed abruptly.

\section{Experiments}

\subsection{Materials}

The asymmetric double cantilever beam (ADCB) specimens consisted of an FRP sheet bonded to the rectangular concrete block with an epoxy adhesive. The concrete consisted of a mixture of water, cement, sand and aggregate (with a mean particle size of $10 \mathrm{~mm}$ ) in the ratio of 0.68: 1: 2: 3 by mass, which was mixed using a mixer for ten min before casting in the mould. After three days, the concrete blocks were taken out and post-cured in an environmental chamber at $27^{\circ} \mathrm{C}$ and $80 \% \mathrm{RH}$ for 28 days. The average compressive strength of concrete was 35.7 MPa, which was obtained from the compressive tests of cylindrical specimens of $100 \mathrm{~mm}$ in diameter and $200 \mathrm{~mm}$ in height.

The carbon fiber reinforced plastic (CFRP) sheet used in this work (MRL-T7200, supplied by Reno Carbon Fiber) was a unidirectional carbon fiber reinforced epoxy prepreg with a nominal tensile strength of $4.2 \mathrm{GPa}$ and a modulus of $235 \mathrm{GPa}$. The adhesive used to bond the CFRP onto the concrete consisted of an epoxy resin (MRLA3), a primer (MRL-A2) and a hardener (MRL-B2). The primer for bond enhancement 
was a mixture of epoxy primer and hardener, and the resin was a mixture of epoxy resin and hardener, both in the ratio of 100:35 by weight.

\subsection{Specimen fabrication and $A D C B$ test}

The configuration and dimensions of ADCB specimen are shown in Fig. 1. All concrete blocks were $100 \mathrm{~mm} \times 100 \mathrm{~mm}$ in cross section and $250 \mathrm{~mm}$ in length. The concrete block surface was prepared with sand papers. The primer (i.e. a mixture of MRL-A2 and MRL-B2) was applied to cover the concrete surface before the CFRP sheets were bonded using the adhesive (i.e. a mixture of MRL-A3 and MRL-B2). Finally, a resin overcoat was applied on top of the CFRP sheets, followed by curing at room temperature for a week.

After the adhesive was completely cured and the contact surface was cleaned using a sand paper and acetone, a piano hinge was bonded to one end of CFRP sheet using an epoxy (Araldite) (Fig. 1). Two bolts and nuts were also used to ensure firm connection between the piano hinge and the CFRP sheet. Specimen edges were polished and coated with a thin brittle white paint, on which fine lines at $2 \mathrm{~mm}$ intervals were scribed to help locate the advancing crack tip during the test. An initial crack of length $42 \mathrm{~mm}$ was made by placing a Teflon film at an appropriate position during the lay-up of prepregs.

The FRP sheets used were $25 \mathrm{~mm}$ wide, $190 \mathrm{~mm}$ long, and $0.66 \mathrm{~mm}$ thick (with six layers of FRP prepregs). Three different configurations of tapered FRP sheets were considered: i) without taper; ii) with taper terminating at the loading point; and iii) with taper starting from the loading point, as schematically shown in Fig. 2. All tapers were applied in a step-wise manner with a constant taper distance of $50 \mathrm{~mm}$, which is the 
distance between the adjacent FRP layers. The taper distance, $50 \mathrm{~mm}$, was chosen because it was identified as the optimal distance that offered the largest benefits of enhanced load carrying capacity and ductility of the RC beams studied previously. It should be mentioned here that the optimal distance is largely dependent on many materials and geometric parameters, such as the stiffness and thickness of FRP sheets, as well as the concrete property and beam geometry. Therefore, a technique to identify the optimal distance for a practical FRP strengthened RC beam with tapered ends is briefly introduced previously. However, in the interlaminar fracture test, there is no such optimal taper distance. The taper distance chosen here is considered sufficiently long to study the change in interlaminar fracture behaviour while maintaining the total FRP sheet length manageable.

The interlaminar fracture tests were performed at room temperature on a MTS universal testing machine with a $1 \mathrm{kN}$ load cell and under the displacement control mode at a crosshead speed of $2 \mathrm{~mm} / \mathrm{min}$. The crack lengths were monitored, and the corresponding load and displacement were recorded during the interrupted loading and unloading cycles. A total of more than five specimens were tested for a given condition.

\section{Solutions for mixed mode I-II interlaminar fracture toughness}

To obtain the interlaminar crack growth resistance curves, it is necessary to calculate the critical strain energy release rate, $G_{c}$, as a function of crack length. A solution was formulated in our previous study [11] of the strain energy release rate for crack propagation along the bi-material interface [12] in the ADCB specimen. In this paper, the critical strain energy release rates, $G_{c}$, was determined based on the compliance method [13]: 


$$
G_{c}=\frac{P^{2}}{2 B} \frac{d C}{d a}
$$

where the compliance $C=\delta / P$. $\delta$ and $P$ are the displacement and the instantaneous load, respectively. $B$ and $a$ are the width of FRP sheet and crack length, respectively. Displacement, $\delta$, was determined using the equations derived in two different approaches: namely the analytical compliance method according to the simple beam theory and the empirical compliance method [14].

In the analytical compliance method, the critical strain energy release rate, $G_{c}$, of the ADCB specimens without taper is calculated as a function of $\delta, P$ and $a$, which is basically the same as that used previously [11]:

$$
\begin{gathered}
\delta=\frac{P a^{3}}{3 E I} \\
G_{c}=\frac{3 P \delta}{2 B a} .
\end{gathered}
$$

The changes in thickness of the FRP sheets in both cases of the taper terminating at the loading point (Fig. 3(a)) and the taper starting from the loading point (Fig. 3(b)) were taken into account in three stages. For a crack shorter than $L_{1}$ (i.e. within Region I), Eqs. (2) and (3) are valid. When the crack is longer than the initial taper distance of $L_{1}$, (i.e. the crack tip located within Region II), the thickness and the second moment of inertia will change from $h_{1}$ to $h_{2}$, and from $I_{1}$ to $I_{2}$, respectively. The corresponding displacement, $\delta$, is a function of second moment and crack length:

$$
\delta=\frac{P L_{1}^{3}}{3 E I_{1}}+\frac{P\left(a-L_{1}\right)^{2}}{6 E I_{2}}\left(2 a+L_{1}\right)+\frac{P\left(a^{2}-L_{1}{ }^{2}\right)}{2 E I_{2}} L_{1} .
$$

Therefore, combining Eqs. (1) and (4) gives $G_{c}$ (for crack tip located within Region II): 


$$
G_{c}=\frac{P a^{2} \delta}{B} \frac{1}{\frac{2}{3} \frac{L_{1}^{3} I_{2}}{I_{1}}+\frac{1}{3}\left(a-L_{1}\right)^{2}\left(2 a+L_{1}\right)+\left(a^{2}-L_{1}{ }^{2}\right) L_{1}}
$$

Once the crack further propagates beyond the second taper distance, $L_{2}$, (i.e. the crack tip located within Region III) the thickness and the second moment of inertia will change from $h_{2}$ to $h_{3}$, and from $I_{2}$ to $I_{3}$, respectively. The displacement, $\delta$, is now given by:

$$
\begin{aligned}
& \delta=\frac{P L_{1}{ }^{3}}{3 E I_{1}}+\frac{P L_{2}{ }^{3}}{3 E I_{2}}+\frac{P L_{1} L_{2}{ }^{2}}{E I_{2}}+\frac{P L_{1}{ }^{2} L_{2}}{E I_{2}}+\frac{P\left(a-L_{1}-L_{2}\right)^{2}}{E I_{3}}\left(\frac{2 a+L_{1}+L_{2}}{6}\right) \\
& +\frac{P\left(L_{1}+L_{2}\right)\left(a-L_{1}-L_{2}\right)}{E I_{3}}\left(\frac{a+L_{1}+L_{2}}{2}\right)
\end{aligned}
$$

and the corresponding equation for $G_{c}$ in Region III becomes:

$$
G_{c}=\frac{P a^{2} \delta}{B} \frac{1}{\left[\begin{array}{l}
\frac{2}{3} \frac{L_{1}^{3} I_{3}}{I_{1}}+\frac{I_{3}}{3 I_{2}} L_{2}{ }^{2}\left(3 L_{1}+2 L_{2}\right)+\frac{I_{3}}{I_{2}} L_{2} L_{1}\left(2 L_{1}+L_{2}\right)+\frac{2\left(a-L_{1}-L_{2}\right)^{3}}{3} \\
+2 a\left(L_{1}+L_{2}\right)\left(a-L_{1}-L_{2}\right)
\end{array}\right]}
$$

In the empirical calibration method, the displacement, $\delta$, is given [14]:

$$
\delta=\frac{P a^{n}}{H}
$$

Combining Eqs. (1) and (8), the final equation for $G_{c}$ can be obtained:

$$
G_{c}=\frac{n P \delta}{2 B a} .
$$

\section{Results and Discussion}

\subsection{Verification of solutions for displacement and deformation}

To be able to use the solutions developed above with confidence, it was necessary to verify the accuracy of displacements described by Eqs. (2), (4) and (6). It was suspected 
that the local, abrupt changes in stiffness at the cut-off points of taper might give rise to an abnormal deformation of the cantilever beam during testing, in particular when the crack tip was located in Region III. Fig. 4 presents the FEM models corresponding to the two extreme cases where the crack tip is located in Region III in both cases with tapers terminating at the loading point and starting from the loading point. The far end of the FRP sheet was fixed, and the relative nodes were tied to the boundary. Eight-node plane stress elements were used for the mesh generation, and the FRP material was assumed to be isotropic and linear elastic. The width of FRP sheets was $25 \mathrm{~mm}$, which is the same as the specimens used in experiments. For samples with a taper terminating at the loading point, the FRP sheet thickness was varied from $4.5 \mathrm{~mm}, 3 \mathrm{~mm}$ to $1.5 \mathrm{~mm}$, with the corresponding lengths of $94 \mathrm{~mm}, 50 \mathrm{~mm}$ and $28 \mathrm{~mm}$, respectively, for Regions I, II and III. For samples with a taper starting from the loading point, the FRP sheet thickness was varied from $1.5 \mathrm{~mm}, 3 \mathrm{~mm}$ to $4.5 \mathrm{~mm}$, with the corresponding lengths of $90 \mathrm{~mm}, 50 \mathrm{~mm}$ and $12 \mathrm{~mm}$, respectively, for Regions I, II and III. Applied loads of 5.8 $\mathrm{N}$ and $24.24 \mathrm{~N}$ were measured from the experiment when the crack lengths were 172 $\mathrm{mm}$ and $152 \mathrm{~mm}$, respectively, for the above two cases. The same loads were applied to the respective FRP sheets in the FEM analysis to analyse the deformations in the FRP sheet, which are compared with those calculated based on the analytical equations and the photographs taken during experiment, as shown in Figs. 5 and 6. It is noted that for both cases of taper design, the displacement profiles along the FRP sheet obtained under the same load from three different methods were essentially the same, in particular between the analytical and FEM calculations. The deformations were perfectly continuous along the whole cantilever even at the taper positions where the FRP sheet thickness changed abruptly. This partly confirms the validity of the ADCB test that was 
used to measure the mixed mode I-II interlaminar fracture toughness of tapered FRP sheets from concrete. The displacement profiles along the FRP sheet were not measured in this experiment. Further quantitative comparisons of the maximum displacement obtained at the loading point are made with reference to Figure 7 in the following.

Fig. 7 presents the comparison of maximum displacements at the loading point plotted as a function of crack length, which was predicted based on the FEM simulation and analytical equations or measured from experiments. There was a clear difference between the maximum displacement data for the two cases of taper. For the taper terminating at the loading point, the maximum displacement increased approximately parabolically with respect to crack length, showing its slope gradually increasing (Fig. 7(a)), whereas for the taper starting from the loading point, the corresponding slope decreased with increasing crack length (Fig. 7(b)). Further, both data presented significant stepwise variations along the crack length with apparent discontinuities, in particular those obtained from the FEM and theoretical analyses. These observations reflected the changes in beam compliance due to the abrupt variations of FRP thickness in a stepwise manner, which influenced more predominantly the displacement data than the increasing crack length. Marginal disparities between the experimental data and the FEM/analytical predictions were also noted, which may be attributed partly to the isotropic, linear elastic material assumptions and partly to the inaccurate measurements of crack lengths. To incorporate the stepwise changes in maximum displacement in three different regions, three different exponent, $n$, values corresponding to different FRP sheet thicknesses were used to calculate the strain energy release rate based on Eq. (8). 


\subsection{Load-displacement curves}

The typical load-displacement curves recorded during the interlaminar fracture test with different taper conditions are shown in Fig. 8. The taper configuration affected significantly the interlaminar fracture resistance behaviour. When the FRP sheet thickness was uniform without taper (Fig. 2a), the critical load decreased gradually with crack growth along the interface (Fig. 8a). When there was a taper terminating at the loading point (Fig. 2b), the load dropped precipitously to a low value at the initial stage of crack propagation, which was followed by rather stable crack growth at much slowly changing load values (Fig. 8b). Precipitous load drops occurred when the crack suddenly propagated beyond the first and second taper positions where the FRP thicknesses suddenly decreased. Therefore, the load-displacement curve clearly presented three stages at different load levels corresponding to three different FRP sheet thicknesses. When the taper was made from the loading point (Fig. 2c), a reversed trend was observed in terms of load-displacement record (Fig. 8c). When the crack propagated across the taper position where the FRP sheet thickness suddenly increased, the critical load did not decrease, but it rather increased to a certain extent due to the increase in FRP sheet thickness.

One of the major advantages of using tapered FRP sheets was that the crack propagation becomes more stable than without. This was manifested by the maximum displacement corresponding to the load drop to zero after complete delamination, which was much higher for the samples with taper, especially for those with taper starting from loading point (Fig. 8c), than without. 


\subsection{Effect of taper on interlaminar fracture toughness}

Figs. 9 and 10 show the critical strain energy release rates measured as a function of crack length for three taper configurations, using the analytical and the empirical compliance methods, respectively. The multiple data points for each condition represent the results for multiple samples. It is noted that the $G_{c}$ values calculated using the analytical compliance method were slightly higher than those calculated using the empirical compliance method. Nevertheless, both methods predicted basically the similar general trend of strain energy release rates vs crack length for the three conditions with and without tapers.

When the FRP sheet thickness was uniform without taper, $G_{c}$ of the FRPadhesive-concrete interface decreased gradually with increasing crack length (Figs. 9a and 10a). As opposed to the interlaminar crack growth behaviour of many polymeric adhesives and laminate composites $[15,16]$, the critical energy release rate of the FRPadhesive-concrete interface with non-tapered FRP sheets varied irregularly, or even tended to decrease with increasing crack length. A similar irregular fracture behaviour was reported previously [11,17] for similar material combinations consisting of CFRP layers bonded to the concrete with an epoxy adhesive. The irregular fracture toughness variation was a reflection of the macroscopically inhomogeneous nature of concrete due to the presence of coarse sand, aggregates and voids, and the crack propagation through the concrete slightly away from the adhesive-concrete interface (i.e. cohesive failure of concrete). Fig. 11 presents a photograph showing a typical fracture surface. This observation held for all samples regardless of whether the FRP sheets had tapers or not. 
When the FRP taper was terminated at the loading point, the $G_{c}$ increased and dropped abruptly when the crack passed the taper points (Figs. 9b and 10b). These abrupt surges and drops at the taper points reflected the sudden changes in effective stiffness or FRP sheet thickness. Careful observations of the fracture surface revealed that crack propagation was not completely uniform across the width of the FRP sheets, but was rather irregular, due to the random presence of coarse sand, aggregates and voids. This made the measurements of instantaneous crack length inaccurate. There was another factor making the measurement of crack lengths difficult: the crack propagation became always rapid when it was closer to the taper position. As a result, the sudden surges and drops of $G_{c}$ were shown rather exaggerated, but in reality there should be relatively smaller changes in $G_{c}$.

For the case of the taper starting from the loading point, $G_{c}$ decreased consistently with increasing crack length (Figs. 9c and 10c), similarly to the results for the samples without taper. Nevertheless, the initial $G_{c}$ values at small crack lengths were much higher than the samples without taper. This observation was partly because the thickness close to the loading point was only a third of that corresponding to the samples without taper, which favourably gave rise to $G_{C}$ of tapered samples although the critical loads were much lower than the samples without taper.

\section{Concluding remarks}

The interlaminar fracture resistance of FRP sheet-concrete interface was evaluated using the asymmetric double cantilever beam (ADCB) test. Solutions were formulated based on both the analytical and empirical compliance methods to determine the critical strain 
energy release rates taking into account the varying thickness of FRP sheets. Tapers were applied in a step-wise manner with a constant taper distance of $50 \mathrm{~mm}$.

The results showed that the taper configuration could significantly affect the load-displacement behaviour as well as the crack growth resistance curves. The loaddisplacement curves clearly presented three stages with different load levels corresponding to three different FRP sheet thicknesses, which were not present for samples without taper. The tapered FRP sheets resulted in more stable crack propagation than those without, due to the enhanced maximum displacement for complete delamination of tapered FRP sheets.

The critical strain energy release rates, $G_{c}$, vs crack length showed characteristic surges or drops for the FRP sheets with taper terminating from the loading point, reflecting the changes in FRP sheet thickness at the points of taper. With the FRP sheets with taper starting from the loading point, the initial $G_{c}$ value at small crack lengths were even higher than the samples without taper.

\section{Acknowledgements}

The Research Grants Council of the Hong Kong SAR (Project No. HKUST 6050/99E), provided the financial support of this work. The authors wish to thank the Construction Materials Laboratory and Advanced Engineering Material Facilities in HKUST for their technical supports. 


\section{References}

[1] Chaallal O, Nollet M-J, Perraton D. Strengthening of reinforced concrete beams with externally bonded fiber-reinforced-plastic plates: design guidelines for shear and flexure. Can J Civ Eng 1998; 25: 692-704.

[2] Saadatmanesh H, Malek AM. Design Guidelines for Strengthening of RC Beam with FRP Plates. J Compos Constr 1998; 2(4): 158-164.

[3] Newman A. Structural Renovation of Building, McGraw-Hill Book Co. New York. 2001.

[4] Teng JG, Chen JF, Smith ST, Lam L. FRP-Strengthened RC Structures, John Wiley \& Sons, Chichester. 2002.

[5] Teng JG, Zhang JW, Smith ST. Interfacial stresses in reinforced concrete beams bonded with a soffit plate: a finite element study. Constr Build Mat 2002; 16: 1-14.

[6] Pesic N, Pilakoutas K. Concrete beams with externally bonded flexural FRPreinforcement: analytical investigation of debonding failure. Compos: Part B 2003; 34: 327-338.

[7] Pham H, Al-Mahaidi R. Assessment of available prediction models for the strength of FRP retrofitted RC beams. Compos Struc 2004; 66: 601-610.

[8] Gao B, Kim JK and Leung CKY. Fracture behaviour of RC beams with FRP sheets bonded with rubber modified resins: experiment and FEM model. Compos. Sci. Technol. 2004; 64: 2557-2564.

[9] Smith ST, Teng JG. FRP-strengthened RC beams. I: review of debonding strength models. Eng Struct 2002; 24: 385-395.

[10] Smith ST, Teng JG. FRP-strengthened RC beams. II: assessment of debonding strength models. Eng Struct 2002; 24: 397-417. 
[11] Gao B, Kim JK, Leung CKY. Effect of rubber modifier on interlaminar fracture toughness of CFRP-concrete interface. Compos Sci Technol 2003; 63: 883-892.

[12] Hutchineson JW. Mixed mode cracking in layered materials. Adv Appl Mech 1992; 29: 163-191.

[13] Friedrich K. Application of fracture mechanics to composite materials. Elsevier Science Publishers BV, Amsterdam. 1989.

[14] Berry JP. Determination of fracture surface energies by the cleavage technique. J Appl Physics 1963; 34(1).

[15] Garg AC, Mai YW. Failure mechanisms in toughened epoxy resins-a review. Compos Sci Technol 1988; 31: 179-223.

[16] Kim JK, Baillie C, Poh J, Mai YW. Fracture toughness of CFRP with modified epoxy matrices. Compos Sci Technol 1992; 43: 283-297.

[17] Kimpara I, Kageyama K, Suzuki T, Osawa I, Yamaguchi K. Characterisation of debonding energy release rate of FRP sheets bonded on mortar and concrete. Adv Compos Mater 1999; 8: 177-187. 


\section{Figure Captions}

FIG. 1: Configuration and dimensions of ADCB specimen for interlaminar fracture test. FIG. 2: Three taper configurations of ADCB specimens including (a) no taper, (b) taper terminating at the loading point and (c) taper starting from the loading point. (Units in $\mathrm{mm})$

FIG. 3: Schematics of ADCB specimens showing three regions where the crack tip locates for (a) taper terminating at the loading point and (b) taper starting from the loading point.

FIG. 4: Two FEM models for verifying the equations of displacement for (a) taper terminating at the loading point and (b) taper starting from the loading point.

FIG. 5: Displacements of tapered FRP sheet plotted as a function of distance from the crack tip for taper terminating at the loading point: (a) FEM model, (b) analytical equations and (c) photograph taken from experiment.

FIG. 6: Displacements of tapered FRP sheet plotted as a function of distance from the crack tip for taper starting from the loading point: (a) FEM model, (b) analytical equations and (c) photograph taken from experiment.

FIG. 7: Comparison of maximum displacements at the loading point plotted as a function of crack length for (a) taper terminating at the loading point and (b) taper starting from the loading point.

FIG. 8: Typical load-displacement curves of interlaminar fracture test for three cases including (a) no taper, (b) taper terminating at the loading point and (c) taper starting from the loading point.

FIG. 9: Strain energy release rate vs crack length curves using analytical compliance method for three cases including (a) no taper, (b) taper terminating at the loading point and (c) taper starting from the loading point.

FIG. 10: Strain energy release rate vs crack length curves using empirical compliance method for three cases including (a) no taper, (b) taper terminating at the loading point and (c) taper starting from the loading point.

FIG. 11: Photograph of a typical fracture surface. 


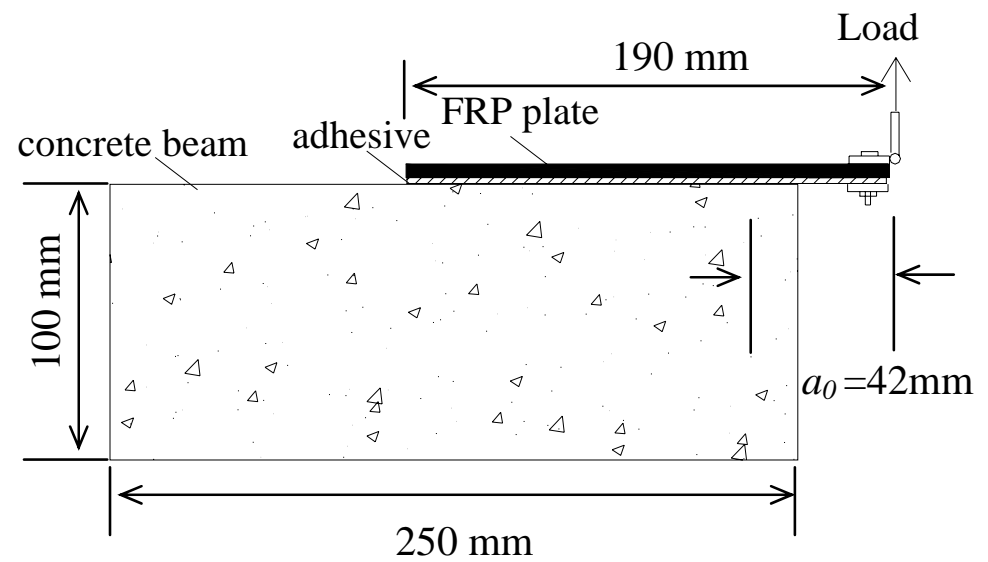

Fig. 1. Configuration and dimensions of ADCB specimen for interlaminar fracture test. 


\section{Load}

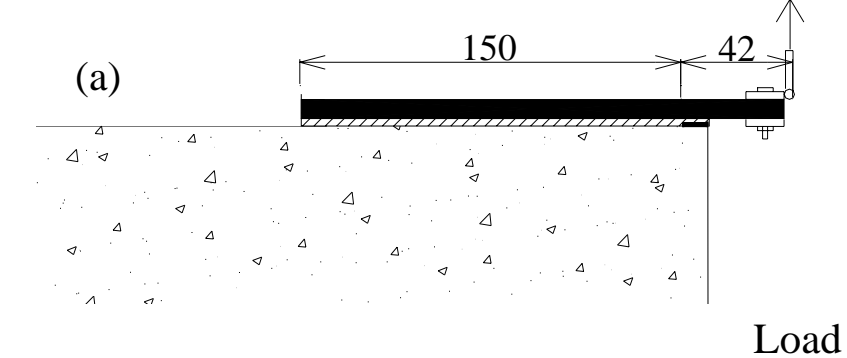

(b)

${ }^{50}+50 \div 50>$

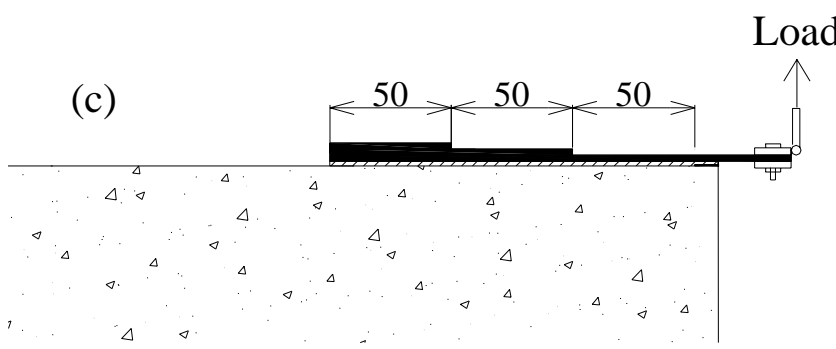

Fig. 2. Three taper configurations of ADCB specimens including (a) no taper, (b) taper terminating at the loading point and (c) taper starting from the loading point. (Units in $\mathrm{mm})$ 


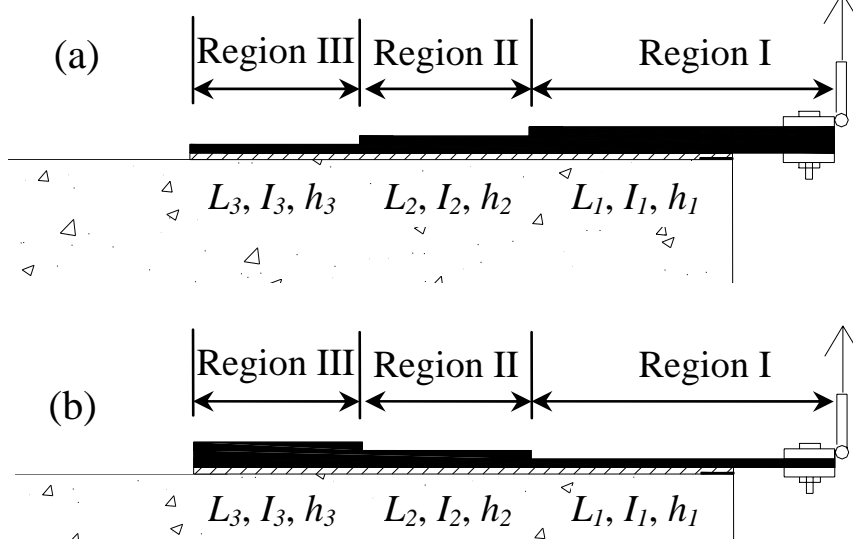

Fig. 3. Schematics of ADCB specimens showing three regions where the crack tip is located for (a) taper terminating at the loading point and (b) taper starting from the loading point. 
(a)

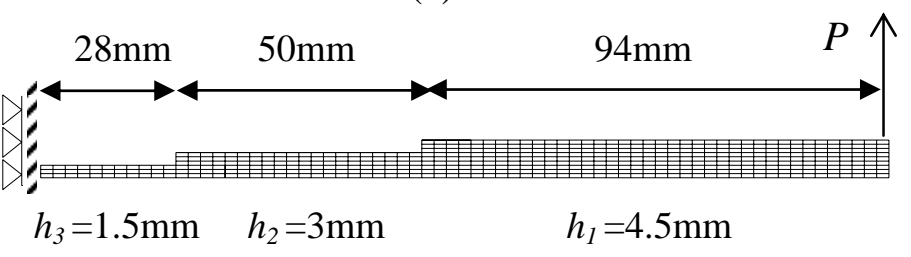

(b)

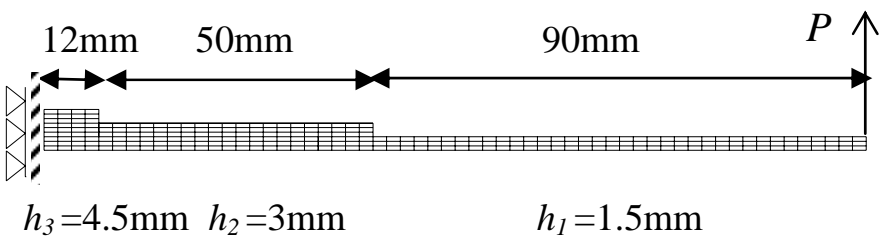

Fig. 4. FEM models used to analyse the loading-point displacements for (a) taper terminating at the loading point and (b) taper starting from the loading point. 


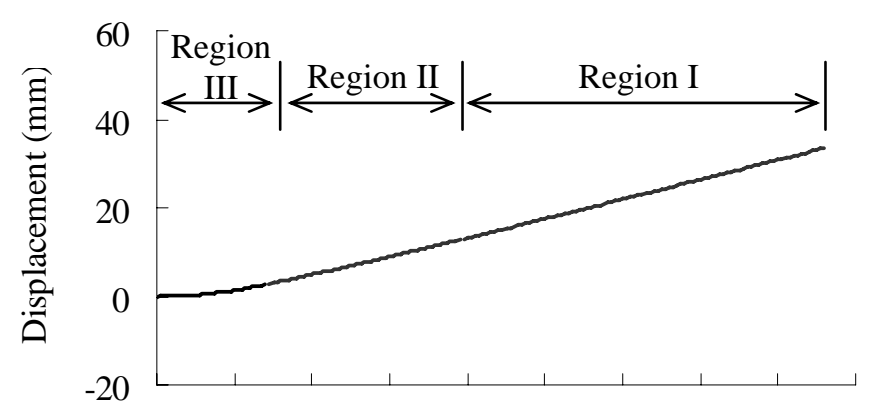

(a) $\quad \begin{array}{llllllllll}0 & 20 & 40 & 60 & 80 & 100 & 120 & 140 & 160 & 180\end{array}$

Distance from the crack tip (mm)

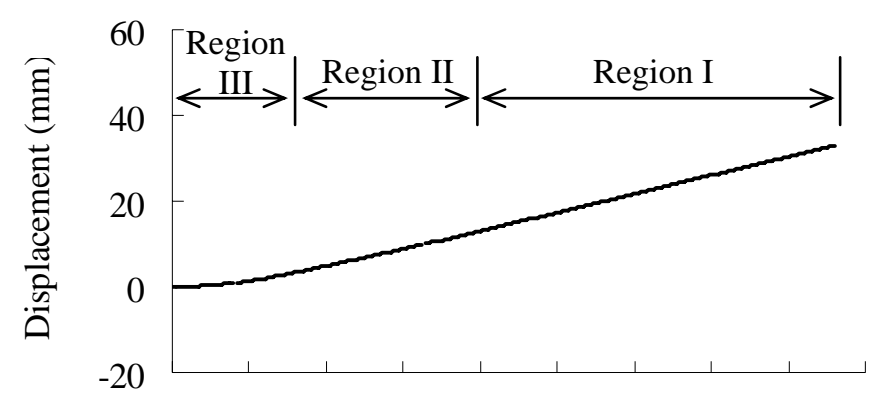

(b) $\quad \begin{array}{llllllllll}0 & 20 & 40 & 60 & 80 & 100 & 120 & 140 & 160 & 180\end{array}$ Distance from the crack tip (mm)

(c)

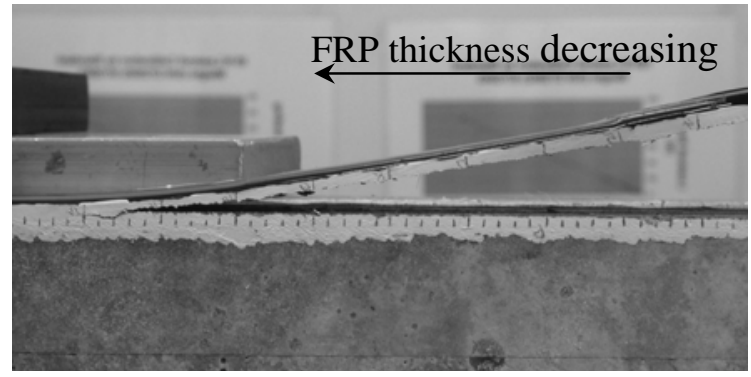

Fig. 5. Displacements of tapered FRP sheet plotted as a function of distance from the crack tip for taper terminating at the loading point: (a) FEM model, (b) analytical equations and (c) photograph taken from experiment. 


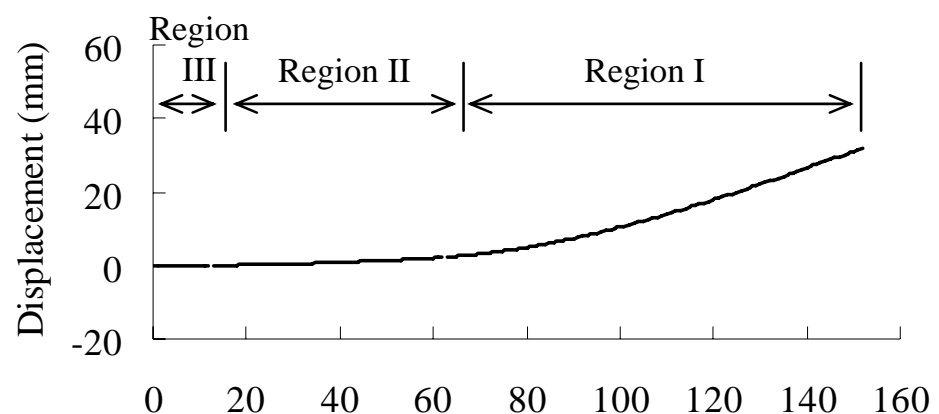

(a) Distance from the crack tip (mm)

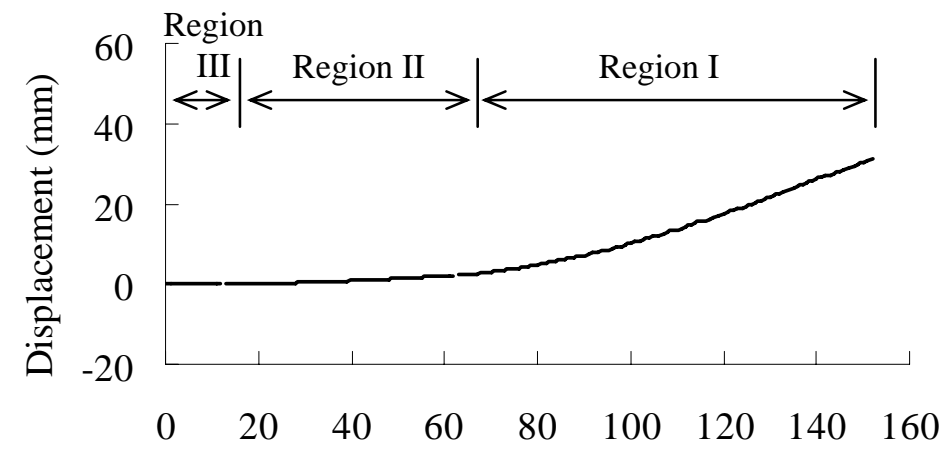

(b) Distance from the crack tip (mm)

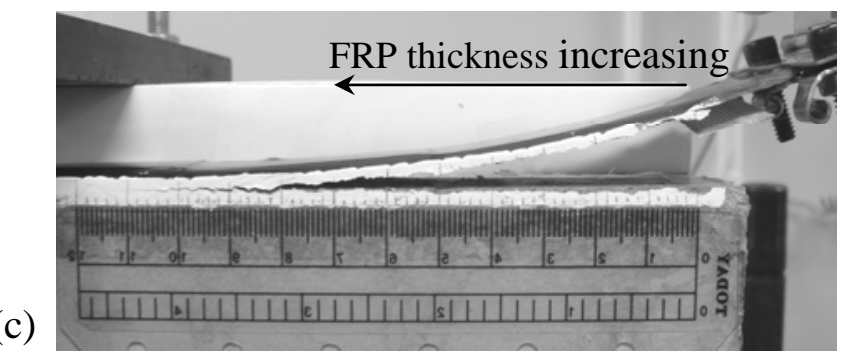

Fig. 6. Displacements of tapered FRP sheet plotted as a function of distance from the crack tip for taper starting from the loading point: (a) FEM model, (b) analytical equations and (c) photograph taken from experiment. 

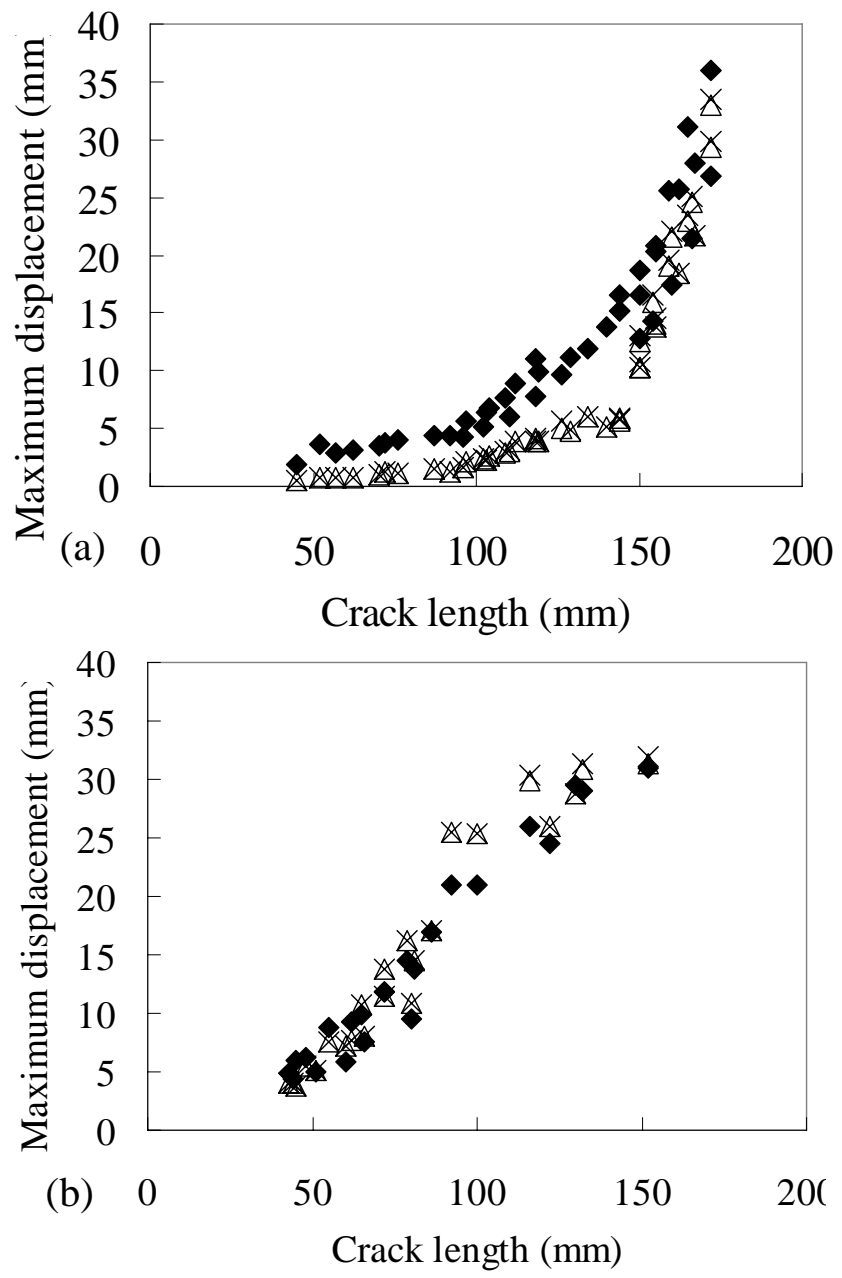

- Experimental measurement

$\triangle$ Prediction by analytical equation $\times$ Prediction by FEM model

Fig. 7. Comparison of maximum displacements at the loading point plotted as a function of crack length for (a) taper terminating at the loading point and (b) taper starting from the loading point. 

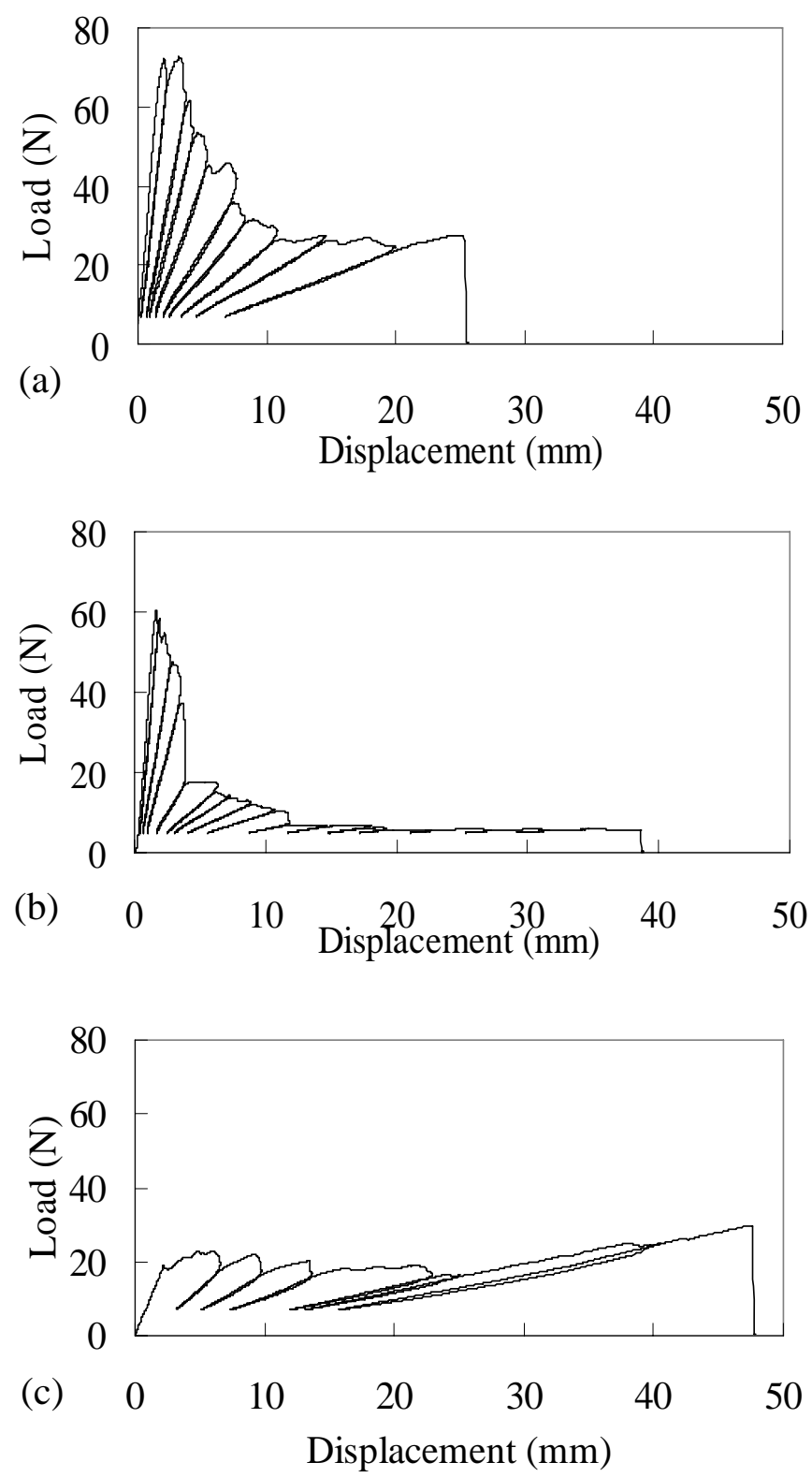

Fig. 8. Typical load-displacement curves of interlaminar fracture test for three cases including (a) no taper, (b) taper terminating at the loading point and (c) taper starting from the loading point. 

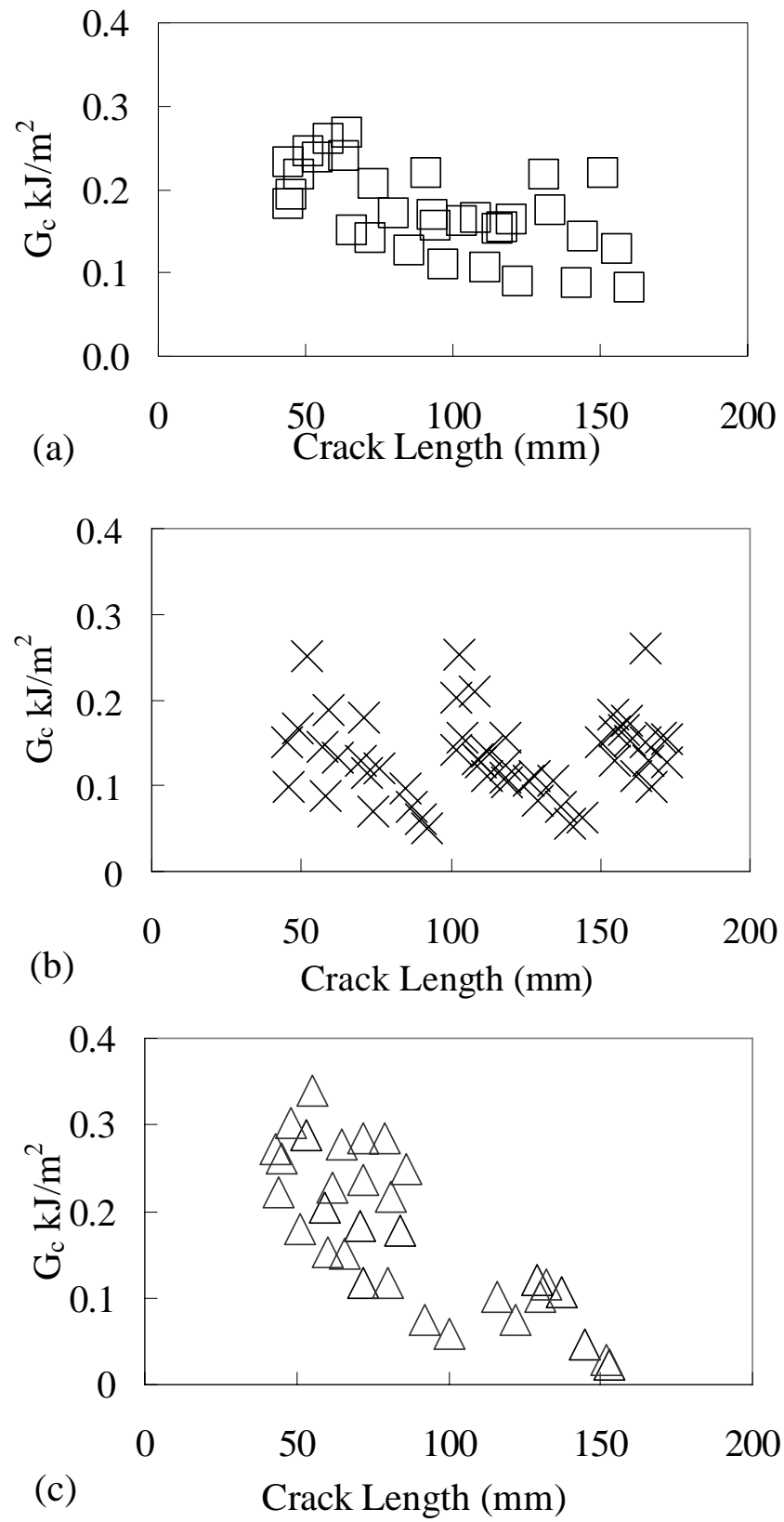

Fig. 9. Strain energy release rate vs crack length curves using analytical compliance method for three cases including (a) no taper, (b) taper terminating at the loading point and (c) taper starting from the loading point. 

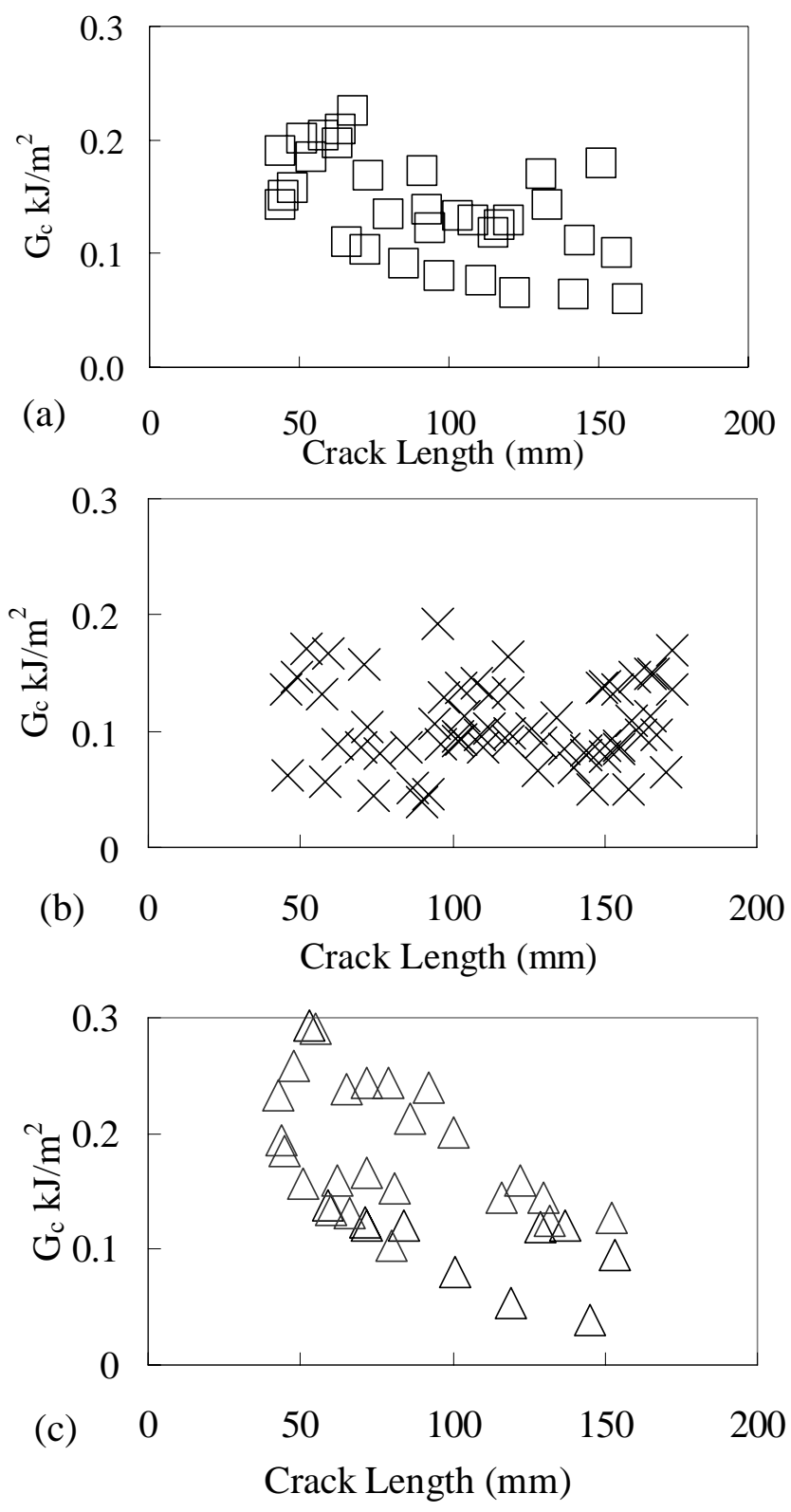

Fig. 10. Strain energy release rate vs crack length curves using empirical compliance method for three cases including (a) no taper, (b) taper terminating at the loading point and (c) taper starting from the loading point. 


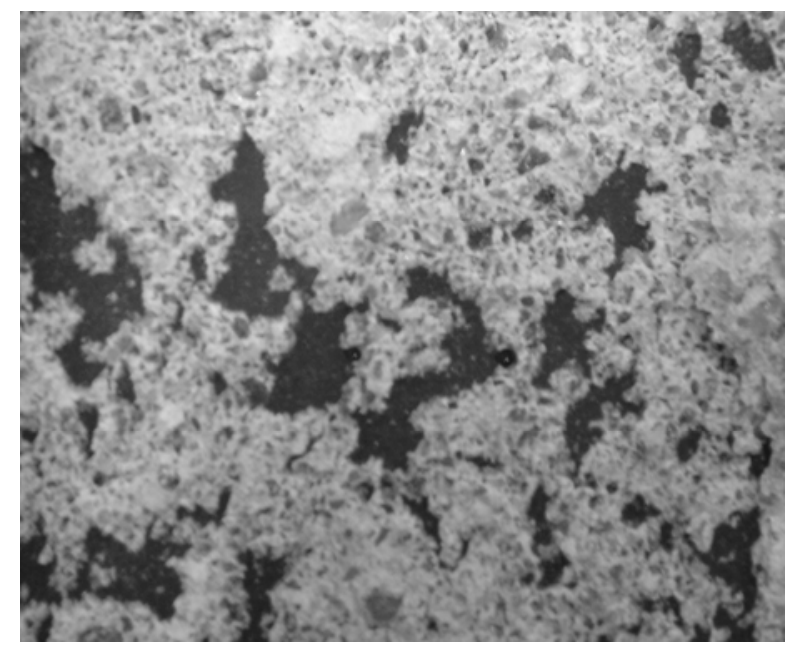

Fig. 11. Photograph of a typical fracture surface. 